\title{
ARBORIZAÇÃO DAS PRAÇAS DE GURUPI - TO - BRASIL: COMPOSIÇÃO E DIVERSIDADE DE ESPÉCIES
}

\author{
AFFORESTATION OF THE SQUARES IN GURUPI-TO-BRAZIL: COMPOSITION AND \\ DIVERSITY OF SPECIES
}

\begin{abstract}
Allan Deyvid Pereira da Silva ${ }^{1}$, Antonio Carlos Batista², Marcos Vinícius Giongo ${ }^{3}$, Daniela Biondi4 André Ferreira dos Santos ${ }^{5}$, Lucicléia Mendes de Oliveira ${ }^{6}$, Jader Nunes Cachoeira ${ }^{7}$.
\end{abstract}

\section{RESUMO}

As praças desempenham relevante papel socioambiental no cenário urbano e sua arborização é um patrimônio que deve ser conhecido e conservado. O objetivo deste estudo foi inventariar as espécies arbóreas das 16 praças de Gurupi - TO, identificá-las quanto a origem, bem como, determinar a diversidade de espécies. Foram inventariadas, com identificação a nível de espécie, todas as árvores e palmeiras pertencentes as praças do município. Dessa forma, constatou-se que a arborização das praças de Gurupi possui1386 indivíduos, 26 famílias, 91 espécies, 66\% são nativas e 34\% exóticas da flora brasileira, a cidade tem alta diversidade de espécies $\left(H^{\prime}=4,77\right.$ e $D^{\prime}=0,93$ ), e o Oiti (Licania tomentosa Benth. Fritsch) é a espécie dominante (18,54\%). As praças que apresentaram os maiores índices de diversidade de espécies foram as praças Waldir Lins $\left(H^{\prime}=3,66\right.$ e $\left.D^{\prime}=0,88\right)$, Praça da Bíblia $\left(H^{\prime}=3,5\right.$ e $\left.D^{\prime}=0,86\right)$ e João Borges Leitão $\left(H^{\prime}=3,43\right.$ e $\left.D^{\prime}=0,88\right)$, e as que apresentaram os menores índices de diversidade foram as praças Vila Íris $\left(H^{\prime}=1,43\right.$ e $\left.D^{\prime}=0,47\right)$ e José Leandro da Silva $\left(H^{\prime}=1,86\right.$ e D' $=0,50$ ).

Palavras-chave: Áreas verdes; Floresta urbana; Fitossociologia.

\section{ABSTRACT}

Squares play a socio-environmental role in urban landscape and their urban trees are a patrimony that must be known and conserved. The aim of this study was to inventory the arboreal species of 16 squares in Gurupi, classify them in exotic or native of Brazilian flora, as well as, to determinate the diversity of species. An inventory was carried out with identification at species level of all trees and palm trees belonging to the 16 public squares of Gurupi municipality, state of Tocantins. In this way, it was found that the Gurupi squares have 1386 individuals, 26 families, 91 species, $66 \%$ are native and $34 \%$ are exotic of Brazilian flora, the city has high species diversity $\left(H^{\prime}=4,77\right.$ e $\left.D^{\prime}=0,93\right)$, and Licania tomentosa is the dominant specie $(18,54 \%)$. The squares that show larger indexes of species diversity were Waldir Lins $\left(H^{\prime}=3,66\right.$ e D' $\left.=0,88\right)$, Biblia $\left(H^{\prime}=3,5\right.$ e D' $\left.=0,86\right)$ and João Borges Leitão $\left(H^{\prime}=3,43\right.$ e D' $\left.=0,88\right)$, and those that show the minors diversity indices were Vila Íris $\left(H^{\prime}=1,43\right.$ e $\left.D^{\prime}=0,47\right)$ and José Leandro da Silva $\left(H^{\prime}=1,86\right.$ e $\left.D^{\prime}=0,50\right)$.

Keywords: Green areas; Urban forest; Phytosociology.

Recebido em 21.06.2019 e aceito em 22.10.2019

1 Geógrafo. Doutorando. Universidade Federal do Paraná. Curitiba/PR. Email: allanuft@gmail.com

2 Eng. Florestal. Doutor. Professor titular da Universidade Federal do Paraná. Curitiba/PR. Email: batistaufpr@gmail.com

3 Eng. Florestal. Doutor. Professor titular da Universidade Federal do Tocantins. Gurupi/TO. Email: giongo@uft.edu.br

4 Eng. ${ }^{\text {a }}$ Florestal. Doutora. Professora Titular da Universidade Federal do Paraná. Curitiba/PR. Email:

danielabiondibatista@gmail.com

5 Eng. Florestal. Doutor. Professor Titular da Universidade Federal do Tocantins. Gurupi/TO. Email: andrefs@mail.uft.edu.br

6 Bióloga. Doutora. Professora da SEDUC da Paraíba. João Pessoa/PB. Email: lucicleiabiologa@gmail.com

7 Bacharel em Direito. Doutorando. Universidade Federal do Paraná. Curitiba/PR. Email: jadercachu@gmail.com 


\section{INTRODUÇÃO}

A praça pública, desde o seu surgimento, apresenta-se como uma área de grande importância para as atividades de inter-relação social, portanto, trata-se de uma importante ferramenta histórica e cultural urbana que expressa as características singulares do desenvolvimento de inúmeras cidades, sendo reflexo direto do processo de urbanização (BENINI; MARTIN, 2010).

Ainda segundo os mesmos autores, no Brasil, as praças públicas foram introduzidas logo nos primeiros séculos da colonização e ficaram marcadas pelo seu potencial de valoração da paisagem, aglomeração de pessoas e por consequência tornaram-se palco de atividades políticas, militares, administrativas, culturais, religiosas, econômicas e de lazer social.

Historicamente, a praça tem se apresentado como um instrumento que contribui para a melhoria da qualidade de vida das pessoas, à medida que apresenta as funções social, ecológica, estética e educativa. Essas funções amenizam as consequências negativas da urbanização (TUAN, 2012).

Considerando a importante contribuição das praças para a inter-relação das sociedades urbanas, recomenda-se a estruturação desses espaços para oferecer maior qualidade ambiental no meio urbano. Dessa forma, as praças devem conciliar a influência do desenvolvimento arquitetônico urbano com a conservação ecológica e ambiental, fazendo com que seja proporcionada a melhor qualidade de vida à população urbana (TUAN, 2012; OLIVEIRA et al., 2013).

Um dos principais elementos que caracterizam a qualidade ecológica e ambiental de uma praça é a sua arborização. A arborização contribui para a melhoria da qualidade do ar, água, solos, clima, ameniza os efeitos do calor provocado pelo aquecimento dos pavimentos e eleva a umidade do ar devido à evapotranspiração. Assim, a arborização urbana é um dos mais importantes componentes dos ecossistemas urbanos, por isso deve ser incluída em todos os planejamentos urbanos de forma sistematizada (OLIVEIRA et al., 2013).

Um dos fatores mais importantes no manejo das florestas urbanas é a promoção da sua heterogeneidade. A maior diversidade de espécies diminui o risco de dizimação de muitos indivíduos de uma mesma espécie por um agente danoso específico e além de contribuir para o paisagismo com um ambiente esteticamente mais harmonioso, traz benefícios aos frequentadores e atrai mais visitantes (SANTAMOUR JR, 2002).

Neste sentido, o conhecimento do patrimônio arbóreo de praças públicas por meio de inventário é essencial para conhecer as condições desses ambientes e detectar possíveis necessidades de manejo. Dessa forma, os índices de diversidade florística apresentam-se como importantes indicadores das condições ecológicas das praças e parques, podendo ser usados como ferramenta de manejo para elaboração ou aperfeiçoamento do plano diretor da 
arborização urbana (KRAMER; KRUPER, 2012; SUBBURAYALU; SYDNOR, 2012; BIONDI; BOBROWSKI, 2014; ZHINENG et al., 2016).

A diversidade de espécies compreende os conceitos de riqueza e de uniformidade. Sendo a riqueza representa pelo número total de espécies em uma área específica e a uniformidade refere-se ao grau de dominância de uma ou mais espécies em uma determinada área (KRAMER; KRUPER, 2012; SUBBURAYALU; SYDNOR, 2012; BIONDI; BOBROWSKI, 2014; ZHINENG et al., 2016).

Os índices de diversidade são utilizados em diversas pesquisas como uma ferramenta de identificação das características da composição dos povoamentos florestais e alguns dos mais utilizados são os de diversidade propostos por Shannon (H') e Simpson (D') e o de uniformidade proposto por Pielou (J) (KRAMER; KRUPER, 2012; SUBBURAYALU; SYDNOR, 2012; BIONDI; BOBROWSKI, 2014; ZHINENG et al., 2016).

Considerando todos esses pressupostos e entendendo a importância da qualidade da arborização em espaços públicos, sobretudo nas praças, este trabalho teve como objetivo: inventariar as espécies arbóreas das 16 praças de Gurupi - TO, identificá-las em exóticas ou nativas da flora brasileira, bem como, determinar a diversidade de espécies.

\section{MATERIAL E MÉTODOS}

A área de estudo compreende as 16 praças públicas do município de Gurupi, Tocantins, Brasil. A sede municipal está localizada entre as coordenadas $11^{\circ} 43^{\prime}$ 45"'S de latitude e $49^{\circ} 04^{\prime}$ '07'O de longitude, com um território de 1.836,091 km² e altitude média de $287 \mathrm{~m}$, situada no sudoeste do estado do Tocantins (Figura 1).

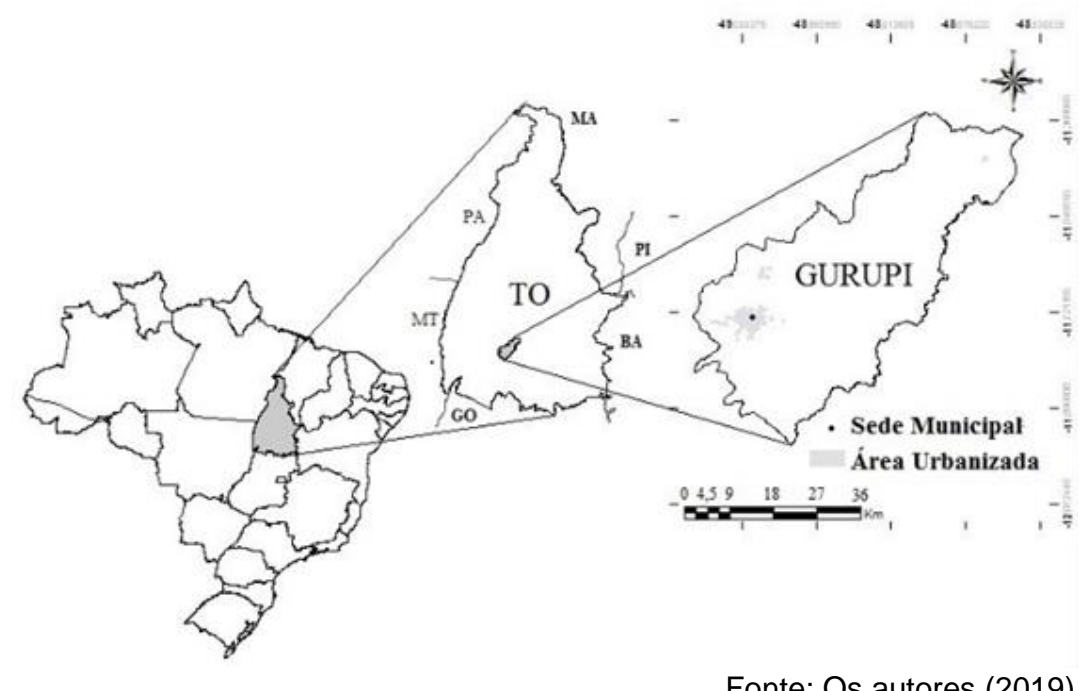

Figura 1. Município de Gurupi - TO e sua área urbana

Fonte: Os autores (2019)

Figure 1. Gurupi municipality - TO and its urban area 
De acordo com a classificação climática de Thornthwaite, o clima em Gurupi é do tipo C2wA'a", clima úmido subúmido com moderada deficiência hídrica no inverno, evapotranspiração potencial média anual de $1.500 \mathrm{~mm}$, distribuindo-se no verão em $420 \mathrm{~mm}$ ao longo dos três meses consecutivos com temperatura mais elevada (TOCANTINS, 2012).

Os solos do município são predominantemente do tipo Latossolos, Plintossolos e Argissolos, contudo, a área urbana encontra-se em uma região com predominância de Argissolos (TOCANTINS, 2012).

Gurupi está localizado nas regiões fitoecológicas do Cerrado, com formações vegetais campestres, savânicas e florestais (TOCANTINS, 2012).

De acordo com o IBGE (2019) Gurupi possuía em 2010, ano do último censo, uma população de 76.755 habitantes (a estimativa para 2019 é de 86.647 habitantes), sendo 75.000 residentes na zona urbana e 1.755 na zona rural, o município apresenta ainda densidade demográfica de $41,80 \mathrm{hab} / \mathrm{km}^{2}$.

A cidade possui 16 praças as quais foram o objeto de estudo deste trabalho. Durante os meses de agosto, setembro e outubro de 2016 foi inventariada a arborização das praças públicas do município e o critério de inclusão de indivíduos considerou espécies arbóreas com circunferência a altura da base maior ou igual a $3 \mathrm{~cm}$, separando-as por família botânica, nome científico e origem (nativa ou exótica da flora brasileira).

A identificação se deu por meio de exsicatas, bibliografia específica (SOUZA; LORENZI, 2005; LORENZI, 2008, 2009a, 2009b), com o auxílio de especialistas e do herbário de Porto Nacional - TO.

Calculou-se a frequência dos indivíduos inventariados, obtendo-se a porcentagem por espécie e família em relação ao total de indivíduos pesquisados, assim como para as espécies agrupadas em nativas e exóticas.

De acordo com Kramer e Kruper (2012), foi calculado o índice de diversidade de Shannon-Wiener:

$$
H^{\prime}=\sum_{i=1}^{S}(p i)(\log p i)
$$

Onde: H' - índice de diversidade proposto por Shannon, pi - abundância relativa de cada espécie, sendo $\mathrm{pi}=\mathrm{ni} / \mathrm{N}$, em que ni - abundância de cada espécie do tipo i e $\mathrm{N}$ - número total de indivíduos coletados, sendo $S$ - número total de espécies amostradas. E pelo critério de Magurran (1988), os valores do índice normalmente estão entre 1,5 e 3,5 nats/ind-1.

De acordo com Subburayalu e Sydnor (2012), foi calculado o índice de diversidade proposto por Simpson:

$$
D^{\prime}=1-\sum_{i=1}^{s}(p i)^{2}
$$


Onde: D' - índice de diversidade de Simpson; pi - abundância relativa (proporção) da espécie i na amostra e $S$ - número de espécies amostradas. $O$ índice de Simpson varia de 0 a 1 e à medida que $\mathrm{D}$ se aproxima de 1 eleva-se a diversidade da população.

O índice de uniformidade ou equabilidade proposto por Pielou foi calculado de acordo com a metodologia descrita em Biondi e Bobrowski (2014), considerando-se o número total de espécies e o índice de diversidade de Shannon:

$$
I=\frac{H^{\prime}}{H m a ́ x}
$$

Onde: $\mathrm{J}$ - equabilidade proposta por Pielou, $\mathrm{H}^{\prime}$ - índice de diversidade proposto por Shannon, Hmáx = log (S); sendo S - número total de espécies amostradas. O intervalo de variação é de 0 a 1 , onde 1 representa a máxima diversidade, ou seja, todas as espécies são igualmente abundantes.

\section{RESULTADOS E DISCUSSÃO}

Nas 16 praças estudadas foram identificados 1386 exemplares arbóreos, pertencentes a 26 famílias botânicas e 91 espécies, dentre estas, 60 são nativas da flora brasileira e 31 exóticas (Tabela 1).

Tabela 1. Tabela 1. Indivíduos arbóreos inventariados nas praças púbicas de Gurupi, TO, Brasil (2016), em função da família, nome científico, número total de indivíduos (NTI), frequência (F) e origem das espécies (O) em nativas (N1) ou exóticas (E2)

Table 1. Tree individuals inventoried in the public squares of Gurupi, TO, Brazil (2014), in function of the family, scientific name, number of individuals (NTI), frequency $(F)$ and origin of species $(O)$ in native (N1) or exotic (E2) species

\begin{tabular}{|c|c|c|c|c|}
\hline Nome Popular & Família/Nome Científico & NTI & F (\%) & $\mathbf{0}$ \\
\hline & Anacardiaceae & & & \\
\hline Cajueiro & Anacardium occidentale L. & 42 & 3,03 & $\mathrm{~N}$ \\
\hline Gonçalo-alves & Astronium fraxinifolium Schott ex Spreng. & 10 & 0,72 & $\mathrm{~N}$ \\
\hline Aroeira-branca & Lithraea molleoides (Vell.) Engl. & 1 & 0,07 & $\mathrm{~N}$ \\
\hline Mangueira & Mangifera indica L. & 58 & 4,18 & $\mathrm{E}$ \\
\hline Aroeira-preta & Myracrodruon urundeuva Allemão & 28 & 2,02 & $\mathrm{~N}$ \\
\hline Aroeira & Schinus lentiscifolius Marchand & 1 & 0,07 & $\mathrm{~N}$ \\
\hline Cajá & Spondias mombin L. & 7 & 0,51 & $\mathrm{~N}$ \\
\hline Ciriguela & Spondias purpurea L. & 2 & 0,14 & $\mathrm{~N}$ \\
\hline Imbuzeiro & Spondias tuberosa Arruda & 1 & 0,07 & $\mathrm{~N}$ \\
\hline \multirow[t]{2}{*}{ Imbu-cajá } & Spondias tuberosa Arruda X Spondias mombin L. & 1 & 0,07 & $\mathrm{~N}$ \\
\hline & Annonaceae & & & \\
\hline \multirow[t]{2}{*}{ Pinha } & Annona squamosa $L$. & 6 & 0,43 & $E$ \\
\hline & Apocynaceae & & & \\
\hline Peroba & Aspidosperma subincanum Mart. & 2 & 0,14 & $\mathrm{~N}$ \\
\hline \multirow[t]{2}{*}{ Jasmim-manga } & Plumeria rubra L. & 3 & 0,22 & $E$ \\
\hline & Arecaceae & & & \\
\hline Macaúba & Acrocomia aculeata (Jacq.) Lodd. Ex Martius & 1 & 0,07 & $\mathrm{~N}$ \\
\hline Macaúba-barriguda & Acrocomia intumescens Drude & 5 & 0,36 & $\mathrm{~N}$ \\
\hline Palmeira rabo-de-peixe & Caryota urens L. & 15 & 1,08 & $\mathrm{E}$ \\
\hline Areca-bambu & Dypsis lutescens (H. Wendl.) Beentje \& J. Dransf. & 2 & 0,14 & $\mathrm{E}$ \\
\hline Palmeira leque & Livistona chinensis (Jack.) R. Br. ex. Mart. & 2 & 0,14 & $\mathrm{E}$ \\
\hline Babaçu & Orbignya phalerata Mart & 7 & 0,51 & $\mathrm{~N}$ \\
\hline
\end{tabular}




\begin{tabular}{|c|c|c|c|c|}
\hline Jerivá & Syagrus romanzoffiana (Cham.) Glassman & 1 & 0,07 & $\mathrm{~N}$ \\
\hline Palmeira-de-manila & Veitchia merrillii (Becc) H. E. Moore & 1 & 0,07 & $E$ \\
\hline Dendezeiro & Elaeis guineensis Jacq & 44 & 3,17 & $\mathrm{E}$ \\
\hline Areca-de-lucuba & Dypsis madagascariensis (Becc.) Beentje \& J.Dransf. & 3 & 0,22 & $E$ \\
\hline Tamareira-de-jardim & Phoenix roebelenii O'Brien & 16 & 1,15 & $E$ \\
\hline Palmeira-imperial & Roystonea oleracea (Jacq.) O. F. Cook & 18 & 1,3 & $E$ \\
\hline Pati & Syagrus botryophora Mart. & 1 & 0,07 & $\mathrm{~N}$ \\
\hline \multirow[t]{2}{*}{ Guariroba } & Syagrus oleracea Becc & 118 & 8,51 & $\mathrm{~N}$ \\
\hline & Bignoniaceae & & & \\
\hline Ipê-amarelo & Handroanthus arianeae (A.H. Gentry) S.O. Grose & 1 & 0,07 & $\mathrm{~N}$ \\
\hline Ipê-roxo & Handroanthus avellanedae (Lorentz ex Griseb.) Mattos & 90 & 6,49 & $\mathrm{~N}$ \\
\hline Pau-d'arco & Handroanthus heptaphyllus (Vell.) Mattos & 11 & 0,79 & $\mathrm{~N}$ \\
\hline Ipê-cascudo & Handroanthus vellosoi (Toledo) Mattos & 44 & 3,17 & $\mathrm{~N}$ \\
\hline Ipê-branco-do-brejo & Tabebuia insignis (Miq.) Sandwith & 36 & 2,6 & $\mathrm{~N}$ \\
\hline Ipê-branco & Tabebuia roseoalba (Ridl.) Sandwith & 1 & 0,07 & $\mathrm{~N}$ \\
\hline \multirow[t]{2}{*}{ lpê-de-jardim } & Tecoma stans (L.) Jussieu ex. Kunth & 13 & 0,94 & $E$ \\
\hline & Boraginaceae & & & \\
\hline \multirow[t]{2}{*}{ Claraíba } & Cordia glabrata (Mart.) DC. & 1 & 0,07 & $\mathrm{~N}$ \\
\hline & Caricaceae & & & \\
\hline \multirow[t]{2}{*}{ Mamoeiro } & Carica papaya L. & 2 & 0,14 & $E$ \\
\hline & Caryocaraceae & & & \\
\hline Pegui & Caryocar brasiliense Cambess. & 1 & 0,07 & $\mathrm{~N}$ \\
\hline \multirow[b]{2}{*}{ Embaúba } & Cecropiaceae & & & \\
\hline & Cecropia pachystachya Trécul & 1 & 0,07 & $\mathrm{~N}$ \\
\hline \multirow[b]{2}{*}{ Oiti } & Chrysobalanaceae & & & \\
\hline & Licania tomentosa (Benth.) Fritsch & 257 & 18,54 & $\mathrm{~N}$ \\
\hline \multirow[b]{2}{*}{ Guanandi } & Clusiaceae & & & \\
\hline & Calophyllum brasiliensis Cambess & 1 & 0,07 & $\mathrm{~N}$ \\
\hline & Combretaceae & & & \\
\hline Mirindiba & Buchenavia tomentosa Eichler & 1 & 0,07 & $\mathrm{~N}$ \\
\hline Capitão-do-campo & Terminalia argentea Mart. & 2 & 0,14 & $\mathrm{~N}$ \\
\hline \multirow[t]{2}{*}{ Castanhola } & Terminalia catappa L. & 18 & 1,3 & $E$ \\
\hline & Euphorbiaceae & & & \\
\hline Seringueira & Hevea brasiliensis (Willd. Ex Adr de Juss.) Muell. Arg. & 1 & 0,07 & $\mathrm{~N}$ \\
\hline \multirow[t]{2}{*}{ Nome Popular } & Família/Nome Científico & NTI & $F(\%)$ & 0 \\
\hline & Fabaceae & & & \\
\hline Carolina & Adenanthera pavonina L. & 1 & 0,07 & $E$ \\
\hline Albízia & Albizia lebbeck (L.) Benth. & 2 & 0,14 & $E$ \\
\hline Angico vermelho & Anadenanthera peregrina (L.) Speg. & 30 & 2,16 & $\mathrm{~N}$ \\
\hline Angelim-mirim & Andira fraxinifolia Benth. & 2 & 0,14 & $\mathrm{~N}$ \\
\hline Pata-de-vaca & Bauhinia forficata Link & 34 & 2,45 & $\mathrm{~N}$ \\
\hline Sucupira-preta & Bowdichia virgilioides Kunth & 1 & 0,07 & $\mathrm{~N}$ \\
\hline Cássia-imperial & Cassia fistula $\mathrm{L}$. & 46 & 3,32 & $E$ \\
\hline Sombreiro & Clitoria fairchildiana R. A.Howard. & 2 & 0,14 & $\mathrm{~N}$ \\
\hline Flamboyant & Delonix regia (Bojer ex Hook.) Raf. & 12 & 0,87 & $E$ \\
\hline Tamboril & Enterolobium contortisiliquum (Vell.) Morong & 4 & 0,29 & $\mathrm{~N}$ \\
\hline Ingá-bravo & Lonchocarpus sericeus (Poir.) Kunth ex DC. & 7 & 0,51 & $\mathrm{~N}$ \\
\hline Jacaranda-de-espinho & Machaerium hirtum (Vell.) Stellfeld & 1 & 0,07 & $\mathrm{~N}$ \\
\hline Bálsamo & Myroxylon peruiferum L. f. & 1 & 0,07 & $\mathrm{~N}$ \\
\hline Olho-de-cabra & Ormosia arborea (Vell.) Harms & 1 & 0,07 & $\mathrm{~N}$ \\
\hline Angico-roxo & Parapiptadenia pterosperma (Benth.) Brenan & 2 & 0,14 & $\mathrm{~N}$ \\
\hline Pau-brasil & Paubrasilia echinata Lam. & 1 & 0,07 & $\mathrm{~N}$ \\
\hline Sibipiruna & Poincianella pluviosa (DC.) L.P.Queiroz & 125 & 9,02 & $\mathrm{~N}$ \\
\hline Angico-branco & Senegalia polyphylla (DC.) Britton \& Rose & 2 & 0,14 & $\mathrm{~N}$ \\
\hline Cássia-de-sião & Senna siamea (Lam.) H.S. Irwin \& R.C. Barneby & 1 & 0,07 & $\mathrm{E}$ \\
\hline Tamarindeiro & Tamarindus indica L. & 1 & 0,07 & $E$ \\
\hline \multirow[t]{2}{*}{ Angelim-do-cerrado } & Vatairea macrocarpa (Benth.) Ducke & 1 & 0,07 & $\mathrm{~N}$ \\
\hline & Lamiaceae & & & \\
\hline \multirow[t]{2}{*}{ Tarumã } & Vitex polygama Cham. & 1 & 0,07 & $\mathrm{~N}$ \\
\hline & Malvaceae & & & \\
\hline Pente-de-macaco & Apeiba tibourbou Aubl. & 1 & 0,07 & $\mathrm{~N}$ \\
\hline Mutamba & Guazuma ulmifolia Lam. & 1 & 0,07 & $\mathrm{~N}$ \\
\hline Monguba & Pachira aquatica Aubl. & 17 & 1,23 & $\mathrm{~N}$ \\
\hline Chichá-do-cerrado & Sterculia striata A. St.-Hil. \& Naudin & 2 & 0,14 & $\mathrm{~N}$ \\
\hline
\end{tabular}

\section{Meliaceae}




\begin{tabular}{|c|c|c|c|c|}
\hline Nim & Azadirachta indica A. Juss. & 16 & 1,15 & $E$ \\
\hline Andiroba & Carapa guianensis Aubl. & 3 & 0,22 & $\mathrm{~N}$ \\
\hline Cedro-rosa & Cedrela fissilis Vell. & 1 & 0,07 & $\mathrm{~N}$ \\
\hline Cedro-cheiroso & Cedrela odorata L. & 2 & 0,14 & N \\
\hline Mogno-brasileiro & Swietenia macrophylla King & 4 & 0,29 & $\mathrm{~N}$ \\
\hline \multirow[t]{2}{*}{ Cedro-australiano } & Toona ciliata M. Roem. var. australis (F. v. M.) C. DC & 3 & 0,22 & $E$ \\
\hline & Moraceae & & & \\
\hline Jaqueira & Artocarpus heterophyllus Lam. & 1 & 0,07 & $E$ \\
\hline \multirow[t]{2}{*}{ Ficus } & Ficus benjamina L. & 4 & 0,29 & $E$ \\
\hline & Myrtaceae & & & \\
\hline Eucalipto-cheiroso & Eucalyptus citriodora Hook. $\mathrm{f}$. & 1 & 0,07 & $\mathrm{E}$ \\
\hline Chiclete-vermelho & Eucalyptus ficifolia F. Muell. & 1 & 0,07 & $\mathrm{E}$ \\
\hline Cagaiteira & Eugenia dysenterica DC. & 1 & 0,07 & $\mathrm{~N}$ \\
\hline Pitanga & Eugenia uniflora L. & 1 & 0,07 & $\mathrm{~N}$ \\
\hline Goiabeira & Psidium guajava $\mathrm{L}$. & 41 & 2,96 & $\mathrm{~N}$ \\
\hline \multirow[t]{2}{*}{ Azeitona-preta } & Syzygium cumini (L.) Skeels & 38 & 2,74 & $E$ \\
\hline & Pinaceae & & & \\
\hline Pinheiro & Pinus oocarpa Schiede ex Schltdl. & 4 & 0,29 & $E$ \\
\hline \multirow[t]{2}{*}{ Grevílea } & $\begin{array}{l}\text { Proteaceae } \\
\text { Grevillea robusta A. Cunn. ex. R. Br. }\end{array}$ & 1 & 0,07 & $\mathrm{E}$ \\
\hline & Rubiaceae & & & \\
\hline Jenipapeiro & Genipa americana L. & 11 & 0,79 & $\mathrm{~N}$ \\
\hline \multirow[t]{2}{*}{ Veludo-branco } & Guettarda viburnoides Cham. \& Schltdl. & 1 & 0,07 & $\mathrm{~N}$ \\
\hline & Rutaceae & & & \\
\hline \multirow[t]{2}{*}{ Limoeiro } & Citrus limon (L.) Burm. F & 5 & 0,36 & $\mathrm{E}$ \\
\hline & Sapindaceae & & & \\
\hline \multirow[t]{2}{*}{ Tingui } & Magonia pubescens A. St.-Hil. & 3 & 0,22 & $\mathrm{~N}$ \\
\hline & Verbenaceae & & & \\
\hline Teca & Tectona grandis L.f. & 68 & 4,91 & $E$ \\
\hline Total & Famílias = 25 / Espécies = 91 & 1386 & 100 & - \\
\hline
\end{tabular}

A família botânica que apresenta maior frequência tanto de indivíduos (Figura 2A) quanto de espécies (Figura 2B) foi a Fabaceae, 20 e 22,8\% respectivamente. Essa família é uma das maiores entre as angiospermas com cerca de 18 mil espécies, além disso é a mais utilizada na arborização urbana em todo o país (SOUZA; LORENZI, 2005), em razão da floração e frutificação. Recomenda-se que a frequência de uma família não exceda $30 \%$ da população, para evitar dizimação por pragas e doenças (SANTAMOUR Jr, 2002).

A. Indivíduos por família botânica (\%)
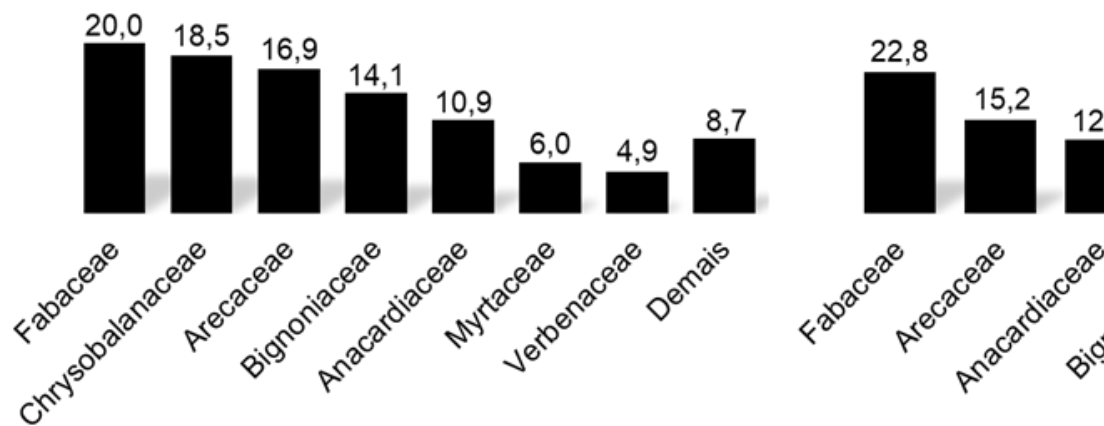

B. Espécies por família botânica (\%)
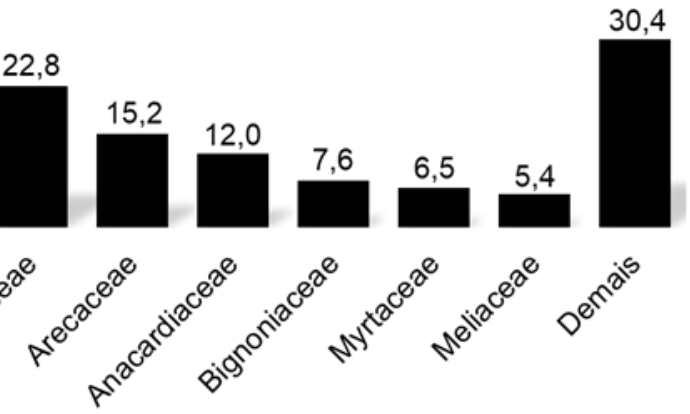

Figura 2. Frequência dos indivíduos (A) e das espécies (B), por família botânica Figure 2. Individuals frequency $(A)$ and species $(B)$ by botanical family 
A família Chrysobalanaceae possui 257 árvores, entretanto, todas são da espécie Oiti (Licania tomentosa), representando $18,5 \%$ do total de indivíduos (Figura 2A). Essa espécie é muito encontrada na arborização urbana em razão de sua rusticidade, copa frondosa, ser perenifólia, além de sua grande resistência às podas (DOS SANTOS; JOSÉ; SOUSA, 2013). No entanto, uma única espécie não deve exceder $10 \%$ da população arbórea, a diminuição de diversidade coloca grande parte da arborização susceptível ao ataque por pragas específicas (SANTAMOUR Jr, 2002), além de promover a perda de qualidade paisagística pela homogeneização da paisagem (MILANO; DALCIN, 2000). Dessa forma, não se recomenda novos plantios de Oiti (Licania tomentosa), podendo esta ser substituída por espécies nativas do Cerrado como o Cega-machado (Physocalymma scaberrimum Pohl) ou o Murici-da-mata (Byrsonima sericea DC.) que aliam beleza cênica e rusticidade nativa.

O número total de espécies encontradas em Gurupi - TO (91) foi semelhante ao verificado em Nova Xavantina - MT (86) e superior ao de Bom Jesus - PI (19) e Caxias - MA (29) (BRITO et al., 2012; LIMA; KREUTZ; PEREIRA, 2016; COSTA et al., 2017).

Para uma boa composição de arborização urbana o número mínimo recomendado são 10 espécies (MILANO; DALCIN, 2000). Neste sentido, para a arborização das praças de Gurupi - TO, que possui um número alto de espécies (91), é indicado que o manejo tenha enfoque maior na distribuição homogênea do número de indivíduos por espécies.

A utilização de espécies exóticas na arborização urbana é um fenômeno comum, seja pelo fator estético, beleza cênica, ou pelo sanitário, ausência de pragas específicas. Todavia, em Gurupi - TO verificou-se um cenário positivo, onde a maior parte das espécies plantadas são nativas da flora brasileira (66\% nativas e $34 \%$ exóticas). Além disso, verificou-se que a taxa de exóticas encontrada nas praças de Gurupi foi inferior à de cidades como Nova Xavantina MT (45\%), Caxias - MA (45\%) e Bom Jesus - PI (70\%) (BRITO et al., 2012; LIMA; KREUTZ; PEREIRA, 2016; COSTA et al., 2017).

Para um cenário ideal, recomenda-se que a arborização seja composta essencialmente por espécies nativas do próprio Bioma em que está inserida a cidade. No caso de Gurupi, espécies nativas do Cerrado são as mais recomendadas, tais como: Ingá (Inga alba (Sw.) Willd.), Sucupira-preta (Bowdichia vigilioides), Chuva-de-ouro (Cassia ferruginea (Schrad.) Schrad. ex DC.), Araribá (Centrolobium tomentosum Guillem. ex Benth.), Faveira (Dimorphandra mollis Benth.), Embiruçu (Pseudobombax longiflorum (Mart. \& Zucc.) A.Robyns), Gomeira (Vochysia thyrsoidea Pohl), Marmelada (Alibertia edulis (Rich.) A.Rich. ex DC.), Pequi (Caryocar brasiliense), Sambaíba (Curatella americana L.), Mulungu (Erythrina speciosa Andrews), Mangaba (Hancornia speciosa Gomes), Pau-santo (Kielmeyera coriácea Mart. \& Zucc.) e Curriola (Pouteria ramiflora (Mart.) Radlk.). Essas espécies apresentam uma 
altura máxima de oito metros, copa densa sem necessidade de poda frequente, além de floração e frutificação restrita a poucos meses no ano (ARAÚJO; PIRES, 2009).

Os índices ecológicos calculados para as praças de Gurupi mostraram a ocorrência de alta diversidade $\left(H^{\prime}=4,77\right.$ e $\left.D^{\prime}=0,93\right)$ e uniformidade de distribuição de espécies $(J=0,73)$ (Tabela 2). O valor de diversidade encontrado em Gurupi foi superior aos das cidades de Cachoeira do Sul - RS com H'= 3,86 e Ribeirão Preto - SP com H'= 3,14 (LINDENMAIER; SANTOS, 2008; ROMANI et al., 2012).

Tabela 2. Praças públicas de Gurupi: número de indivíduos (NI) e de espécies arbóreas (NE) e, seus respectivos índices ecológicos

Table 2. Gurupi public squares: individuals' number (NI) and tree species (NE) and their respective ecological indexes

\begin{tabular}{lllllll}
\hline Nome das praças (Nome Informal) & NI & NE & $\mathbf{H}^{\prime}$ & $\mathbf{D}^{\prime}$ & $\mathbf{J}$ & Área $\mathbf{( m}^{\mathbf{2}} \mathbf{~}$ \\
\hline José Leandro da Silva (Cometa) & 118 & 14 & 1,86 & 0,50 & 0,49 & $7.893,8$ \\
João Borges Leitão (Abadia) & 86 & 17 & 3,43 & 0,88 & 0,84 & $6.029,1$ \\
Praça da Bíblia & 87 & 23 & 3,50 & 0,86 & 0,77 & $5.952,4$ \\
Praça da Rodoviária & 42 & 13 & 2,98 & 0,81 & 0,81 & $11.881,3$ \\
Francisco Henrique Santana (Centro Cultural) & 142 & 17 & 2,70 & 0,75 & 0,66 & $11.164,2$ \\
Praça do Sol & 93 & 19 & 3,29 & 0,85 & 0,77 & $3.912,0$ \\
Praça do Tadeu & 54 & 12 & 2,72 & 0,77 & 0,76 & $1.575,0$ \\
Praça Pauliceia & 81 & 16 & 3,00 & 0,82 & 0,75 & $5.588,4$ \\
Praça Pedro Dias & 41 & 9 & 2,77 & 0,82 & 0,87 & $1.024,3$ \\
Praça Santo Antônio & 110 & 22 & 2,95 & 0,78 & 0,66 & $10.536,7$ \\
Praça São Lucas & 56 & 15 & 3,12 & 0,83 & 0,80 & $3.721,5$ \\
Praça Sol Nascente & 79 & 12 & 2,33 & 0,73 & 0,65 & $10.693,5$ \\
Praça Trevo Sul & 114 & 18 & 2,99 & 0,79 & 0,72 & $5.164,1$ \\
Praça Vila Íris & 14 & 5 & 1,43 & 0,47 & 0,62 & 807,4 \\
Praça Vila Nova & 12 & 7 & 2,69 & 0,83 & 0,96 & $1.758,3$ \\
Praça Waldir Lins & 257 & 28 & 3,66 & 0,88 & 0,76 & $21.649,8$ \\
\hline Geral & 1386 & 91 & 4,77 & 0,93 & 0,73 & - \\
\hline
\end{tabular}

Nota: H' - Índice de Shannon; D' - Índice de Simpson; J - Índice de Pielou; a informação de área $\left(\mathrm{m}^{2}\right)$ foi consultada em Da Silva, Dos Santos e De Oliveira (2016).

A diversidade de espécies mensurada individualmente, por praça, demonstrou que as praças Waldir Lins ( $H^{\prime}=3,66$ e D' = 0,88), Praça da Bíblia $\left(H^{\prime}=3,5\right.$ e D' = 0,86) e João Borges Leitão ( $H^{\prime}=3,43$ e D' = 0,88) são as que possuem a maior diversidade de espécies (Tabela 2).

As praças da Bíblia e João Borges Leitão estão entre as mais velhas do município com árvores e palmeiras bem estabelecidas, onde houve notadamente um planejamento de implantação da arborização.

$\mathrm{Na}$ Praça Waldir Lins, em virtude do pouco tempo desde sua criação, foram introduzidas muitas mudas de diversas espécies (DA SILVA; DOS SANTOS; DE OLIVEIRA, 2016), o que contribuiu para a alta diversidade verificada na área. Esse tipo de área deve ter um acompanhamento para reposição de indivíduos se necessário for, pois estas plantas jovens apresentam pouca resistência física em razão do seu estágio de desenvolvimento e estão 
susceptíveis a uma diminuição de sua população em razão de possíveis depredações (BIONDI; LEAL, 2010).

A Praça Vila Íris teve a menor diversidade $\left(H^{\prime}=1,43\right.$ e $\left.D=0,47\right)$, com apenas cinco espécies e 14 indivíduos inventariados. Contudo, sua área é a menor $\left(807,4 \mathrm{~m}^{2}\right)$ entre todas as praças do município (DA SILVA; DOS SANTOS; DE OLIVEIRA, 2016), o que inviabiliza o plantio de muitas árvores.

A Praça José Leandro da Silva tem o terceiro maior número de indivíduos (118) entre as praças avaliadas e a segunda menor diversidade de espécies $\left(H^{\prime}=1,86\right.$ e $\left.D^{\prime}=0,50\right)$. Essa condição é resultado da baixa distribuição das espécies no local $(\mathrm{J}=0,49)$. Nessa praça recomenda-se a diminuição do número de indivíduos das espécies mais abundantes e a introdução de novas espécies nativas do Cerrado (ARAÚJO; PIRES, 2009).

$\mathrm{Na}$ Praça Vila Nova foram encontrados apenas 12 indivíduos e 7 espécies, mas com boa distribuição $(\mathrm{J}=96)$. De acordo com Da Silva, Dos Santos e De Oliveira (2016), a Praça Vila Íris tem $1.758,3 \mathrm{~m}^{2}$, o que limita o plantio de novos indivíduos.

\section{CONCLUSÕES}

Por meio deste estudo foi possível concluir que a arborização das praças de Gurupi TO possui 1.386 indivíduos, pertencentes a 26 famílias e 91 espécies. Entre as espécies encontradas, $66 \%$ são nativas da flora brasileira, além de apresentarem alta diversidade de espécies $\left(H^{\prime}=4,77\right.$ e $\left.D^{\prime}=0,93\right)$. O Oiti (Licania tomentosa) é a espécie dominante $(18,5 \%)$, sendo necessário cessar o plantio de novos indivíduos desta espécie. As praças que possuem os maiores índices de diversidade de espécies são a Praça Waldir Lins ( $H^{\prime}=3,66$ e D' = 0,88), Praça da Bíblia ( $H^{\prime}=3,5$ e D' = 0,86) e Praça João Borges Leitão $\left(H^{\prime}=3,43\right.$ e D' = 0,88). As que possuem os menores índices de diversidade são as Praças Vila Íris ( $H^{\prime}=1,43$ e D' = 0,47), onde a ausência de espaço dificulta o plantio de maior número de indivíduos, e Praça José Leandro da Silva $\left(H^{\prime}=1,86\right.$ e $\left.D^{\prime}=0,50\right)$, com necessidade de uma substituição gradativa das espécies mais abundantes por novas espécies.

\section{AGRADECIMENTOS}

Agradecemos a Coordenação de Aperfeiçoamento de Pessoal de Nível Superior CAPES, à Universidade Feral do Tocantins - UFT e à Universidade Federal do Paraná UFPR. 


\section{REFERÊNCIAS}

ARAÚJO, R. C. R; PIRES, L. L. Opções de Frutíferas do Cerrado para Paisagismo Urbano em Bairros da Periferia de Goiânia - GO. Revista Caatinga, Mossoró, v. 22, n. 4, p. 235-239, 2009.

BENINI, S. M.; MARTIN, E. S. Decifrando as áreas verdes públicas. Formação, Presidente Prudente, v. 2, n. 17, p. 63-80, 2010.

BIONDI, D.; BOBROWSKI, R. Utilização de índices ecológicos para análise do tratamento paisagístico arbóreo dos parques urbanos de Curitiba-PR. Enciclopédia Biosfera, Jandaia, v. 10, n. 18, p. 3006-3017, 2014.

BIONDI, D.; LEAL, L. Monitoramento de mudas de Allophylus edulis (A. St.-Hil., Cambess. \& A. Juss.) Radlk. Plantadas experimentalmente na arborização de ruas da cidade de Curitiba - PR. Revista da Sociedade Brasileira de Arborização Urbana, Piracicaba, v.5, n.2, p.158-173, 2010.

BRITO, D. R. S.; RAABE, J.; SOUSA, W. C.; MELO, R. R.; PEDROSA, T. D. Diagnóstico da arborização das praças pública no município de Bom Jesus, Piauí. Scientia plena, v. 8, n. 4, p. 1-6, 2012.

COSTA, C.; FONSECA, R.; ALMEIDA, D.; OLIVEIRA, M.; OLIVEIRA, D.; BRAGA, J. Espécies utilizadas na arborização em praças do município de Caxias, Maranhão. Revista da Sociedade Brasileira de Arborização Urbana, Piracicaba, v. 12, n. 1, p. 65-78, 2017.

DA SILVA, A. D. P.; DOS SANTOS, A. F.; DE OLIVEIRA, L. M. Índices de área verde e cobertura vegetal das praças públicas da cidade de Gurupi, TO. Floresta, Curitiba, v. 46, n. 3, p. 353-362, 2016.

DOS SANTOS, A. F.; JOSÉ, A. C.; SOUSA, P. A. Fitossociologia e diversidade de espécies arbóreas das praças centrais do município de Gurupi-TO. Revista da Sociedade Brasileira de Arborização Urbana, Piracicaba, v.8, n.4, p 36- 46, 2013.

INSTITUTO BRASILEIRO DE GEOGRAFIA E ESTATÍSTICA (IBGE).

Brasil/Tocantins/Gurupi. 2019. Disponível em: <https://cidades.ibge.gov.br/brasil/to/gurupi/panorama>. Acesso em: 01 ago. 2019.

KRAMER, J. A.; KRUPEK, R. A. Caracterização florística e ecológica da arborização de praças públicas do município de Guarapuava, PR. Revista Árvore, Viçosa, v. 36, n. 4, p. 647-658, 2012.

LIMA, J. P.; KREUTZ, C.; PEREIRA, O. R. Levantamento florístico das espécies utilizadas na arborização de praças no município de Nova Xavantina-MT. Revista da Sociedade Brasileira de Arborização Urbana, Piracicaba, v. 10, n. 3, p. 60-72, 2016.

LINDENMAIER, D. S.; SANTOS, N. O. Arborização urbana das praças de Cachoeira do Sul, RS, Brasil: fitogeografia, diversidade e índice de áreas verdes. Pesquisas Botânica, São Leopoldo, v. 1, n. 59, p. 307-320, 2008.

LORENZI, H. Árvores brasileiras: manual de identificação e cultivo de plantas arbóreas nativas do Brasil. v. 1, 5. ed. Nova Odessa, SP: Instituto Plantarum, 2008. 
LORENZI, H. Árvores brasileiras: manual de identificação e cultivo de plantas arbóreas nativas do Brasil. v. 2, 3. ed. Nova Odessa, SP: Instituto Plantarum, 2009a.

LORENZI, H. Árvores brasileiras: manual de identificação e cultivo de plantas arbóreas nativas do Brasil. v. 3, 1. ed. Nova Odessa, SP: Instituto Plantarum, 2009b.

MAGURRAN, A.E. Ecological diversity and its measurement. 1. ed. Princeton: Princeton University, 1988. 179p.

MILANO, M.; DALCIN, E. Arborização de vias públicas. 1.ed. Rio de Janeiro: LIGHT, 2000. $226 p$.

OLIVEIRA, A. S.; SANCHES, L.; DE MUSIS, C. R.; NOGUEIRA, M. C. J. A. Benefícios da arborização em praças urbanas - o caso de Cuiabá/MT. Revista Eletrônica em Gestão, Educação e Tecnologia Ambiental, v. 9, n. 9, p. 1900-1915, 2013.

ROMANI, G. N.; GIMENES, R.; SILVA, M. T.; PIVETTA, K. F. L.; BATISTA, G. S. Análise qualiquantitativa da arborização na praça XV de Novembro em Ribeirão Preto - SP, Brasil. Revista Árvore, Viçosa, v.36, n.3, p.479-487, 2012.

SANTAMOUR JR, F. S. Trees for urban planting: diversity uniformity, and common sense. Department of Agriculture Washington, D.C., 2002. Disponível em: <https://pdfs.semanticscholar.org/26a2/4c5361ce6d6e618a9fa307c4a34a3169e309.pdf>. Acesso em: 01 ago.2019.

SOUZA, V. C.; LORENZI, H. Botânica sistemática: guia ilustrado para identificação das famílias de Angiospermas da flora brasileira, baseado em APG II. Nova Odessa, São Paulo: Plantarum, 2005.

SUBBURAYALU, S.; SYDNOR, T. D. Assessing street tree diversity in four Ohio communities using the weighted Simpson index. Landscape and Urban Planning, [S. I.], v. 106, n. 1, p. 4450, 2012.

TOCANTINS. Atlas do Tocantins: subsídios ao planejamento da gestão territorial. Palmas: SEPLAN, 6. ed. rev. atu. 2012.

TUAN, Y. Topofilia: um estudo da percepção, atitudes e valores do meio ambiente. Londrina: EDUEL, 2012.

ZHINENG, L.I.U.; GANG, P. A. N.; HONGFENG, Z. H. A. N. G.; JIANGPING, F. A. N. G.; JIN, X. U.; WEI, W. A. N. G.; PENG, Z. H. O. U. Investigation and Application Research of Landscape Plants in Tibet. Journal of Landscape Research, [S. I.], v. 8, n. 1, p. 60-68, 2016. 